\title{
$K$ Index in cerebrospinal fluid: a valid tool in multiple sclerosis diagnosis
}

\section{Massimo Pieri, Fabio Duranti, Diego Centonze, Fabio Buttari, Sergio Bernardini \& Mariarita Dessì}

La Rivista Italiana della Medicina di Laboratorio - Italian Journal of Laboratory Medicine

ISSN 1825-859X

Riv Ital Med Lab

DOI 10.1007/s13631-014-0072-0

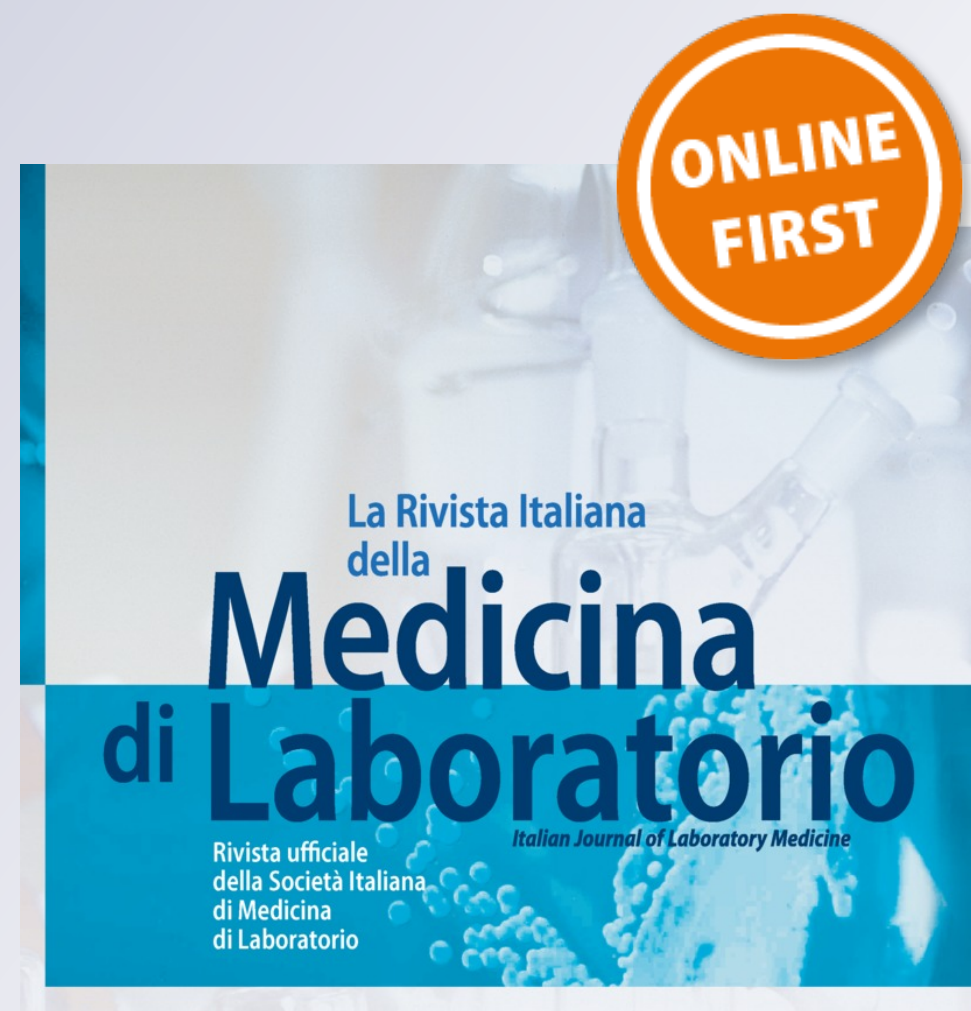

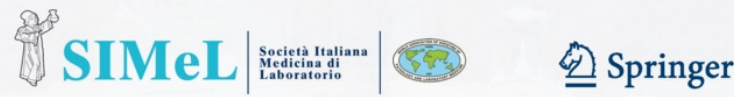


Your article is protected by copyright and all rights are held exclusively by SpringerVerlag Italia. This e-offprint is for personal use only and shall not be self-archived in electronic repositories. If you wish to self-archive your article, please use the accepted manuscript version for posting on your own website. You may further deposit the accepted manuscript version in any repository, provided it is only made publicly available 12 months after official publication or later and provided acknowledgement is given to the original source of publication and a link is inserted to the published article on Springer's website. The link must be accompanied by the following text: "The final publication is available at link.springer.com". 


\title{
K Index in cerebrospinal fluid: a valid tool in multiple sclerosis diagnosis
}

\author{
Massimo Pieri · Fabio Duranti • Diego Centonze • \\ Fabio Buttari · Sergio Bernardini • Mariarita Dessì
}

Ricevuto: 2 febbraio 2014 / Accettato: 9 giugno 2014

(C) Springer-Verlag Italia 2014

\begin{abstract}
Summary Background. Detection of oligoclonal IgG bands in cerebrospinal fluid by isoelectrocfocusing and immunodetection is the current gold standard to detect an inflammatory process in the central nervous system. It has been proposed that the presence of free light chains (FLCS) in CSF was associated with recent demyelination activity in MS and might be used as a prognosis marker. Our study's objective is assessing the diagnostic accuracy of a new highly sensitive latex-enhanced nephelometric assay for $k$ free light chain ( $k F L C$ ) determination in CSF/serum as an alternative to traditional tests and its clinical application.

Methods. kFLCs were measured in CSF/serum pairs from 80 patients by the use of a new highly sensitive latex-enhanced nephelometric automated immunoassay for detection of immunoglobulin FLC. The eighty patients were split into three groups according to the neurological diagnosis. In this study we confirm even more the use of the $k$ Index as a diagnostic aid in multiple sclerosis.

Results. kFLC Index seems to be more accurate parameter respect the determination of oligoclonal immunoglobulin bands (OCBs). We recalculate the $K$ Index sensitivity and specificity respect the precedent published result. Two patients previously diagnosed with leukoencephalopathy have gone to group 3 as confirmed the diagnosis of MS.

Conclusions. These new data reinforce even more the use of the $k$ Index to diagnose MS in comparison to classical methods and to the reference method, the OCBs.
\end{abstract}

\footnotetext{
M. Pieri · F. Duranti · S. Bernardini · M. Dessì $(\varangle)$

Department of Experimental Medicine and Surgery, "Tor

Vergata" University Hospital, Viale Oxford 81, 00133 Roma, Italy

e-mail:mariarita.dessi@uniroma2.it

D. Centonze · F. Buttari

Department of Systems Medicine, "Tor Vergata" University

Hospital \& IRCCS Fondazione Santa Lucia, Roma, Italy
}

Keywords $k F L C s \cdot C S F \cdot$ Nephelometry $\cdot$ OCBs $\cdot k F L C$ Index $\cdot$ Multiple sclerosis

\section{Introduction}

Free Light Chains (FLCs) presence in cerebrospinal fluid (CSF) has been studied in several works regarding neurological disease, especially focusing on Multiple Sclerosis (MS). Up to date mainly used methods comprehend qualitative test such as isoelectrofocusing (IEF) followed by transfer and immunodetection [1-3]. Oligoclonal free light chains banding has been described in MS and should be correlated with disease activity. Intrathecal IgG production may be also present in patients suffering from other central nervous system (CNS) disease, but its detection by current laboratory tests is either tedious or insensitive. Because small amounts of immunoglobulins (Ig) typically enter CSF by passive transfer across the blood/CSF barrier, origin differentiation of Ig in CSF is needed before intrathecal immunoglobulin synthesis can be diagnosed [4]. This can be achieved either by calculation of the CSF/serum ratios of Ig compared with the CSF/serum ratio of albumin (Qalb), which is not synthesized intrathecally, or by the detection of so-called oligoclonal immunoglobulin bands (OCBs) in CSF [5, 6].

Plasma cells produce an excess of kappa and lambda light chains during the production of intact Ig and these are secreted as FLCs. Due to the high fractional excretion and catabolism of the kidneys the half life of FLCs in human serum is only a few hours. Instead in CSF the clearance is slower and even a small increase of kFLCs may be noticeable. Several studies demonstrate the presence of kFLCs in the CSF $[2,7,8]$ and kFLCs may be used as a prognosis marker [8-12]. In this study, we assessed the diagnostic accuracy of an automated nephelometric assay for kFLCs 
in CSF and serum. We compared it with traditional tests to detect intrathecal immunoglobulin synthesis and investigated the usefulness of this method for clinical application. Recently our laboratory investigated CSF/serum pairs from patients: the results obtained are compared with the standard oligoclonal band assessment that was taken as the reference standard for our study [13,14] and demonstrate that the kFLCs Index is a valid tool to MS diagnosis [15]. These results are further improved through a re-evaluation of diagnoses adding to the statistics two cases that had not previously been diagnosed as MS.

\section{Patients}

We test CSF/serum pairs from 80 patients with different clinically well-documented neurological disorders. All patients had undergone an elective spinal puncture at the Neuroscience Department of the Tor Vergata University Hospital. The study protocol complied with the declaration of Helsinki (1964).

Samples were excluded if artificial blood contamination of CSF or monoclonal bands in both CSF and serum were present. According to their diagnoses the patients were divided into three groups: group 1 (33 patients, 21 female and 12 male) patients having diseases without inflammation, patients with inflammatory diseases other than MS group 2 (22 patients, 13 female and 9 male) and patients with definitive MS group 3 ( 25 patients, 16 female and 9 male). Diagnosis of MS was established according to the McDonald criteria [16].

\section{Methods}

OCBs were determined by immunofixation (Hydragel9 CSF Isofocusing; Sebia, Bagno a Ripoli, FI, Italia) on a semiautomated agarose electrophoresis system (Sebia Hydrasys) $[13,14]$. The gels were evaluated for the presence of OCBs by three independent operators.

Nephelometric measurement of kFLCs was performed with the N Latex FLC kappa Kit (Siemens Healthcare Diagnostics Products GmbH, Marburg, Germany) based on monoclonal antibodies, that recognizes hidden epitopes in intact Ig.

FLCs in CSF and serum samples were measured according to the manufacturer's protocol on the BN Prospec automated analyzer (Siemens Healthcare); the lower detection limit for kFLCs is $0.035 \mathrm{mg} / \mathrm{L}$.

Calibrators and controls were provided by the manufacturer and consisted of stabilized human sera containing polyclonal kFLCs; calibrators and controls were diluted to the appropriate concentrations for serum and CSF determinations.
Table 1 Characteristics of the patient groups

\begin{tabular}{|c|c|c|c|}
\hline & $\begin{array}{l}\text { Group 1 } \\
(n=33)\end{array}$ & $\begin{array}{l}\text { Group 2 } \\
(n=22)\end{array}$ & $\begin{array}{l}\text { Group 3 } \\
(n=25)\end{array}$ \\
\hline $\operatorname{Sex}(\mathrm{F} / \mathrm{M})$ & $21 / 12$ & $13 / 9$ & $16 / 9$ \\
\hline $\begin{array}{l}\text { Mean } \\
\text { (minimum-maximum) } \\
\text { age, years }\end{array}$ & $\begin{array}{l}50 \pm 14 \\
(23-79)\end{array}$ & $\begin{array}{l}49 \pm 15 \\
(19-72)\end{array}$ & $\begin{array}{l}38 \pm 11 \\
(21-58)\end{array}$ \\
\hline $\begin{array}{l}\text { Cerebrovascular } \\
\text { disease/bleeding, } n\end{array}$ & 5 & & \\
\hline $\begin{array}{l}\text { Neurodegenerative } \\
\text { diseases, } n\end{array}$ & 7 & & \\
\hline Neoplastic diseases, $n$ & 7 & & \\
\hline Morbus Alzheimer, $n$ & 1 & & \\
\hline $\begin{array}{l}\text { Psychiatric/mental- } \\
\text { disorders, } n\end{array}$ & 5 & & \\
\hline Vertigo, $n$ & 1 & & \\
\hline Focalpartialepilepsy, $n$ & 2 & & \\
\hline Emicrania, $n$ & 2 & & \\
\hline Miateniagravis, $n$ & 2 & & \\
\hline Meningitis, encephalitis, $n$ & & 2 & \\
\hline Leukoencephalopathy, $n$ & & 5 & \\
\hline Cranialneuritis, $n$ & & 2 & \\
\hline Polyneuropathy & & 5 & \\
\hline Myelitis/myelophaties, $n$ & & 7 & \\
\hline Opticneuritis, $n$ & 1 & 1 & \\
\hline MS, $n$ & & & 26 \\
\hline OCBs positive, $n$ & 1 & 4 & 18 \\
\hline IgG Index positive, $n$ & 0 & 2 & 9 \\
\hline
\end{tabular}

$\mathrm{CSF} /$ serum quotients are calculated to evaluate the passively diffusion into the liquor of the FLCs that are also present in serum. An additional factor to consider is the permeability of the blood/CSF barrier. FLCs concentration increases with augmented blood-CSF barrier permeability. The CSF/serum quotient of the kFLCs was therefore plotted against the $Q_{a l b}$, because albumin is only synthesized in liver and diffuses just passively into the CSF. The hyperbolic function commonly used to detect the limit between diffusion and synthesis of FLCs in the CSF is:

$\mathrm{Q}_{\mathrm{FLC}}=\frac{a}{b \sqrt{\left(Q_{a l b}^{2}+b^{2}\right)-c}}$

According to Reiber [17], an optimal fit of the hyperbolic curve may be achieved using the following parameters: $a / b=6.5 ; b^{2} \times 10^{6}=5 ; c \times 10^{3}=1$.

$\mathrm{kFLC}$ Index was calculated as $\frac{\mathrm{kFLC}_{C S F} / \mathrm{kFLC}_{\text {serum }}}{Q_{\text {albumina }}} \times 1000$.

\section{Statistical analysis}

Data were analyzed with Origin 7 (Origin Lab Corporation, Northampton, USA) and SPSS 17.0 (IBM Corporation, New 
Table 2 Statistical results

\begin{tabular}{|c|c|c|c|c|c|}
\hline & & $\mathrm{kFLC}$ liquor, $\mathrm{mg} / \mathrm{L}$ & $\mathrm{kFLC}$ ratio liquor/serum & kFLC Index & IgG Index \\
\hline \multirow{4}{*}{ Group 1} & Median & 0.07 & 0.013 & 2 & 0.47 \\
\hline & Range & $0.04-0.33$ & $0.003-0.045$ & $0.2-8.54$ & $0.13-0.57$ \\
\hline & 25th percentile & 0.058 & 0.0085 & 1.35 & 0.4 \\
\hline & 75th percentile & 0.15 & 0.0195 & 3.25 & 0.5 \\
\hline \multirow[t]{4}{*}{ Group 2} & Median & 0.085 & 0.840 & 3.40 & 0.47 \\
\hline & Range & $0.02-0.32$ & $0.02-0.32$ & $0.50-018.2$ & $0.35-0.60$ \\
\hline & 25th percentile & 0.045 & 0.045 & 2.24 & 0.46 \\
\hline & 75th percentile & 0.18 & 0.18 & 4.60 & 0.49 \\
\hline \multirow[t]{4}{*}{ Group 3} & Median & 1.15 & 0.32 & 41.66 & 0.057 \\
\hline & Range & $0.08-11.7$ & $0.014-2.127$ & $2.7-398.3$ & $0.44-1.57$ \\
\hline & 25th percentile & 0.45 & 0.126 & 20.3 & 0.51 \\
\hline & 75th percentile & 2.87 & 0.65 & 122.36 & 0.90 \\
\hline
\end{tabular}

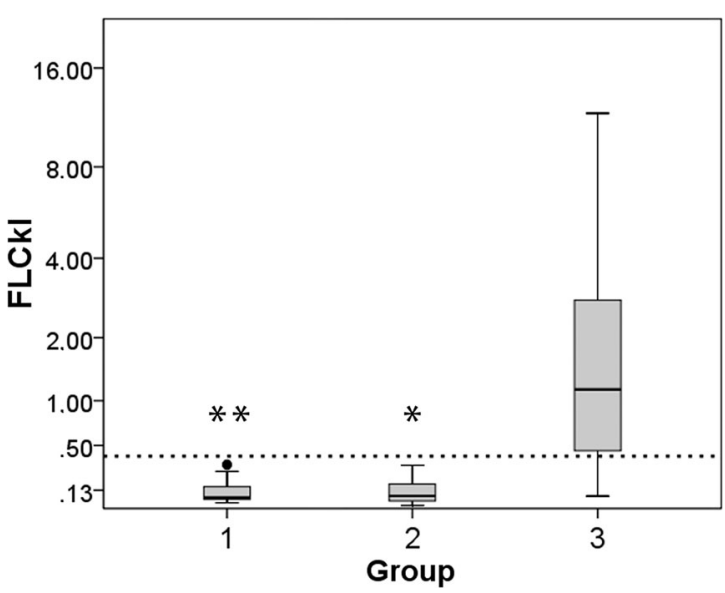

Fig. 1 Box plot of kFLC concentrations (mg/L) in CSF. The median and 25th and 75th percentiles (shaded boxes), the 10th and 90th percentiles (error bars), and the outliers $(\bullet)$ are indicated. Dashed line, cutoff for $\mathrm{kFLC}$ concentrations $(0.4 \mathrm{mg} / \mathrm{L})$. The group 3 is significantly different compared to group $1\left(^{* *} p<0.001\right)$ and group $2\left({ }^{*} p<0.01\right)$

York, USA) software. ANOVA followed by Bonferroni's test was used to determine the significance of the differences between the three groups. A $p$ value of $<0.05$ was considered significant.

\section{Results}

CSF/serum pairs of 80 patients of the neurological clinic were examined. Patient characteristics and patient groups are shown in Table 1.

The median CSF concentration of kFLCs in group 1 was $0.07 \mathrm{mg} / \mathrm{L}$ (range, $0.04-0.33 \mathrm{mg} / \mathrm{L}$ ). $\mathrm{kFLCs}$ were detectable in group 2 , with median of $0.084 \mathrm{mg} / \mathrm{L}$ (range, 0.02 $0.32 \mathrm{mg} / \mathrm{L}$ ) and in group 3 with median of 1.15 (range, 0.08 $11.7 \mathrm{mg} / \mathrm{L}$ ), all statistical values are represented in the Table 2 .

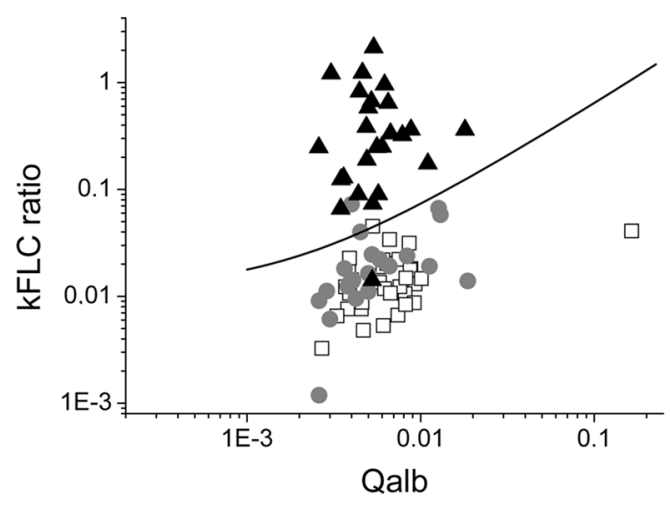

Fig. 2 On the ordinate, cerebrospinal fluid (CSF) serum quotients of free kappa light chains vs albumin CSF serum quotients are plotted logarithmically (quotient diagrams, Reiber plots). Results of the determination of the free kappa light chain concentrations are given. $(\square)$ : group 1 patients; (๑): group 2 patients; $(\mathbf{\Lambda})$ : group 3 patients

All patients from group 1 and group 2, have a kappafree light chain concentrations in CSF below the cutoff of $0.4 \mathrm{mg} / \mathrm{L}$ [15]. All data of the $\mathrm{kFLC}$ concentrations in CSF of the group 3 are above the cut off value and shows a statistically significant difference respect the group 1 and 2 , for details see Figure 1.

kFLCs serum quotients were plotted logarithmically against the albumin CSF serum quotients to determine the influence of the blood CSF barrier (Fig. 2). Using the quotient diagram, intrathecal kappa free light chain production was identified in one patient of group 2. All patients from group 1 lie below the hyperbolic dividing line. Only one patient of group 3 lie below the Reiber function.

The use of kFLC Index calculated as $\frac{\mathrm{kFLC}_{C S F} / \mathrm{kFLC}_{\text {serum }}}{Q_{\text {albumina }}} \times$ 1000 that takes into account the function of the blood/CSF barrier, it's a tentative to increase the diagnostic accuracy of kFLC determination and to better assess the presence of intrathecal immunoglobulin synthesis avoiding false positive. 
The median kFLC Index was 2 (range, 0.2-8.54) for group 1, 3.40 (range, 0.5-18.20) for group 2 and 41.66 (range, 2.69-398.3) for group 3 (Table 2). These are recent data obtained by a new diagnosis of two patients previously diagnosed as leukoencephalitis and revaluated as MS. The two patients are women and have a kFLC Index of 26.6 and 154 respectively, values much higher than the previously published cut-off $>12$ [15].

Finally we correlate all methods of intrathecal immunoglobulin synthesis detection with clinical diagnoses and found new values for clinical sensitivity and specificity as shown in Figure 3.

From our data the calculation of kFLC Index shows the highest clinical sensitivity and specificity (96\% and $91 \%$ respectively) to evaluate MS patients; whereas IgG Index highlights the lowest sensitivity $(60 \%)$ as previously reported in literature [18]. All statistical results are shown in Table 3 .

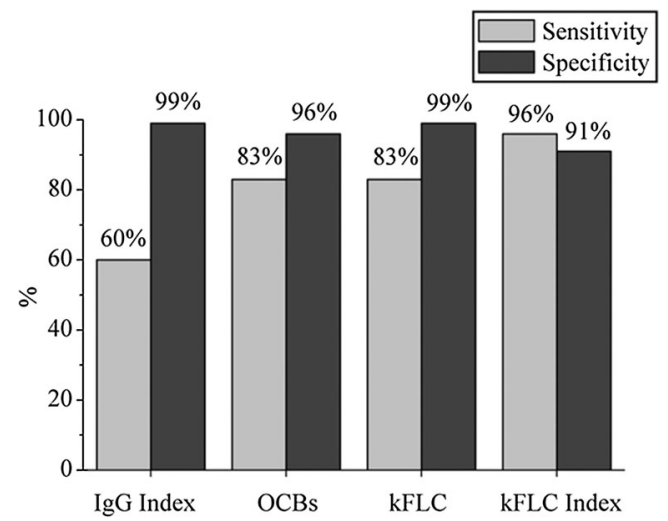

Fig. 3 Clinical sensitivity and specificity using different methods to discriminate MS patients from other neurological disease

\section{Discussion}

In this study, we described the use of the kFLC Index using a highly sensitive latex-enhanced nephelometric automated immunoassay for detection of immunoglobulin kFLC in every paired serum and CSF samples. It has been known for decades that kFLC levels are elevated in the CSF of MS patients $[5,6]$. Development of a sensitive immunoassay for serum or CSF FLC determination has allowed incorporation of FLC analysis into routine laboratory diagnostic algorithms.

We found for kFLC Index a sensitivity of $96 \%$ and a specificity of $91 \%$ for MS diagnosis.

kFLC Index more than lambda FLC index was associated with MS in accordance with previous studies using either qualitative or quantitative methods $[1,10,19]$. We establish that $\mathrm{kFLC}$ index is more sensitive than $\mathrm{IgG}$ index for found intrathecal immune production.

The determination of free immunoglobulin light chains has been technically difficult in the past, operator depending and not feasible in routine clinical diagnostics. Thus, daily assays would be easily integrated into laboratory processes reducing tedious manual procedures and uncertainty in result interpretation. Furthermore, the assay turnaround time is no more than 1.5 hours for this assay, compared with at least 4-5 hours for OCBs determination and can give us a quantification of kFLCs synthesis.

Our previous data [15], has been further improved thanks to a reevaluation of two patients subsequently confirmed with a MS diagnosis. The current study suggest the implementation of kFLC Index determination as a screening method in CSF analysis before further investigation for a possible intrathecal immune response, especially where MS or CIS is suspected.

Our data support even more the use of the kFLC Index to MS diagnose in comparison to the classical methods.
Table 3 Sensitivity, Specificity, Positive and Negative Predictive Values of the methods

\begin{tabular}{|c|c|c|c|c|}
\hline & IgG Index & OCB & kFLC & kFLC index \\
\hline $\begin{array}{l}\text { Sensitivity } \\
\text { (Confidence } \\
\text { interval) }\end{array}$ & $0.60(0.41-0.79)$ & $0.83(0.68-0.97)$ & $0.83(0.69-0.97)$ & $0.96(0.88-1)$ \\
\hline $\begin{array}{l}\text { Specificity } \\
\text { (Confidence } \\
\text { Interval) }\end{array}$ & $0.99(0.96-1)$ & $0.96(0.91-1)$ & $0.99(0.96-1)$ & $0.91(0.83-0.98)$ \\
\hline $\begin{array}{l}\text { Positive } \\
\text { Predictive value } \\
\text { (Confidence } \\
\text { Interval) }\end{array}$ & $1(1-1)$ & $0.90(0.77-1)$ & $1(1-1)$ & $0.96(0.88-1)$ \\
\hline $\begin{array}{l}\text { Negative } \\
\text { Predictive value } \\
\text { (Confidence } \\
\text { Interval) }\end{array}$ & $0.76(0.66-0.86)$ & $0.91(0.83-0.98)$ & $0.91(0.80-1)$ & $0.98(0.94-1)$ \\
\hline
\end{tabular}


These results lead us to introduce kFLC Index test in the CSF diagnostic examinations and to support clinician in a rapid and accurate MS diagnosis.

Conflicts of interest None.

\section{References}

1. Krakauer M, Schaldemose Nielsen H, Jensen J, Sellebjerg F (1998) Intrathecal synthesis of free immunoglobulin light chains in multiple sclerosis. Acta Neurol Scand 98:161-165

2. Lamers KJ, de Jong JG, Jongen PJ et al (1995) Cerebrospinal fluid free kappa light chains versus IgG findings in neurological disorders: qualitative and quantitative measurements. J Neuroimmunol 62:19-25

3. Sindic CJ, Laterre EC (1991) Oligoclonal free kappa and lambda bands in the cerebrospinal fluid of patients with multiple sclerosis and other neurological diseases. An immunoaffinity-mediated capillary blot study. J Neuroimmunol 33:63-72

4. Reiber H, Felgenhauer K (1987) Protein transfer at the blood cerebrospinal fluid barrier and the quantitation of the humoral immune response within the central nervous system. Clin Chim Acta 163:319-328

5. Luxton RW, McLean BN, Thompson EJ (1990) Isoelectric focusing versus quantitative measurements in the detection of intrathecal local synthesis of IgG. Clin Chim Acta 187:297-308

6. Walker RW, Keir G, Johnson MH, Thompson EJ (1983) A rapid method for detecting oligoclonal IgG in unconcentrated CSF, by agarose isoelectric focusing, transfer to cellulose nitrate and immunoperoxidase staining. J Neuroimmunol 4:141-148

7. Lolli F, Amaducci L (1989) Measurement of free kappa immunoglobulin light chains in the cerebrospinal fluid by a competitive avidin-biotin ELISA. Clin Chim Acta 182:229-234

8. Rudick RA, Medendorp SV, Namey M et al (1995) Multiple sclerosis progression in a natural history study: predictive value of cerebrospinal fluid free kappa light chains. Mult Scler 1:150-155
9. Bracco F, Gallo P, Menna R et al (1987) Free light chains in the CSF in multiple sclerosis. J Neurol 234:303-307

10. DeCarli C, Menegus MA, Rudick RA (1987) Free light chains in multiple sclerosis and infections of the CNS. Neurology 37:13341338

11. Freedman MS, Thompson EJ, Deisenhammer F et al (2005) Recommended standard of cerebrospinal fluid analysis in the diagnosis of multiple sclerosis: a consensus statement. Arch Neurol 62:865-870

12. Rudick RA, Cookfair DL, Simonian NA et al (1999) Cerebrospinal fluid abnormalities in a phase III trial of Avonex (IFNbeta-1a) for relapsing multiple sclerosis. The Multiple Sclerosis Collaborative Research Group. J Neuroimmunol 93:8-14

13. Caudie C, Allausen O, Bancel J (2000) Detection of oligoclonal IgG bands in cerebrospinal fluid by immunofixation after electrophoretic migration in the automated Hydrasys sebia system. Ann Biol Clin (Paris) 58:376-379

14. Richard S, Miossec V, Moreau JF, Taupin JL (2002) Detection of oligoclonal immunoglobulins in cerebrospinal fluid by an immunofixation-peroxidase method. Clin Chem 48:167-173

15. Duranti F, Pieri M, Centonze D et al (2013) Determination of kFLC and K Index in cerebrospinal fluid: a valid alternative to assess intrathecal immunoglobulin synthesis. J Neuroimmunol 263:116-120

16. Polman CH, Reingold SC, Banwell B et al (2011) Diagnostic criteria for multiple sclerosis: 2010 revisions to the McDonald criteria. Ann Neurol 69:292-302

17. Reiber H (2001) Dynamics of brain-derived proteins in cerebrospinal fluid. Clin Chim Acta 310:173-186

18. Mares J, Herzig R, Urbanek K et al (2008) Correlation of the IgG index and oligoclonal bands in the cerebrospinal fluid of patients with multiple sclerosis. Biomed Pap Med Fac Univ Palacky Olomouc Czech Repub 152:247-249

19. Fischer C, Arneth B, Koehler J et al (2004) Kappa free light chains in cerebrospinal fluid as markers of intrathecal immunoglobulin synthesis. Clin Chem 50:1809-1813 